\title{
Atividades na formação do terapeuta ocupacional*
}

\section{Activities in the formation of occupational therapist}

\author{
Carla Regina Silva ${ }^{1}$, Jéssica Cristina Von Poellnitz ${ }^{2}$
}

http://dx.doi.org/10.11606/issn.2238-6149.v26i1p74-82

Silva CR, Poellnitz JCV. Atividades na formação do terapeuta ocupacional. Rev Ter Ocup Univ São Paulo. 2015 jan./ abr.;26(1):74-82.

RESUMO: As atividades são compreendidas como elementos centrais na construção complexa e contextualizada do processo terapêutico ocupacional, ressalta-se a importância do seu ensino na formação profissional. Este trabalho apresenta os resultados de uma pesquisa que objetivou mapear e analisar a formação do terapeuta ocupacional, com enfoque no ensino de atividades e recursos oferecido nos cursos de graduação de Instituições de Ensino Superior públicas do Estado de São Paulo. Para tanto, foram utilizados os Projetos Pedagógicos; realizadas entrevistas com coordenadores e aplicados questionários com os docentes envolvidos com o ensino da temática. Foi possível traçar o perfil das instituições em relação ao ensino de atividades, docentes, laboratórios, processos avaliativos, e integração com a pesquisa e a extensão. Concluí-se que o ensino da temática apresenta um núcleo comum, mas ocorre de forma singular nos cursos a depender dos currículos adotados, assim como, da formação e perspectiva dos docentes. Referenda-se a necessidade das atividades se estruturarem como campo próprio de saber e na formação integrar conteúdos teóricos estruturantes aos relacionados à dimensão prática das experimentações e dos fazeres.

DESCRITORES: Atividades e recursos; Terapia ocupacional; Desenvolvimento de pessoal; Ensino, aprendizagem.
Silva CR, Poellnitz JCV. Activities in the formation of occupational therapist. Rev Ter Ocup Univ São Paulo. 2015 jan./abr.;26(1):7482.

\begin{abstract}
The activities are considered central elements in the complex and contextual construction of the occupational therapist process, we emphasize the importance of its teaching in the formation of this professional. We present the results of a research that aimed to map and analyze the formation of occupational therapists, focusing on the teaching activities and the resources offered in the graduation courses of public universities in the São Paulo state. To this end, the Pedagogical Projects and the specific subjects of Activities and Resources were analyzed; the courses coordinators were interviewed and questionnaires were applied to the professors involved in the teaching of this topic. It was possible to trace the profile of the institutions in relation to teaching activities, teachers, laboratories, evaluation processes and integration with research and extension. It is concluded that the teaching of the subject has a common core, but it occurs uniquely in courses depending on the adopted curricula, as well as the training and perspective of the teachers. It endorses the need of activities to structure as a field of knowledge and in the formation to integrate theoretical concepts structuring to the profession to the practical dimension of the doings.
\end{abstract}

KEY WORDS: Activities and resources; Occupational therapy; Staff development; Teaching, Learning.

\footnotetext{
* Artigo apresenta uma síntese da pesquisa de Iniciação Científica "O ensino das atividades e a formação do terapeuta ocupacional" realizada entre agosto de 2012 e agosto de 2013, com apoio da CNPq ProPq UFSCar.

Como parte da concessão de bolsas, parte dos dados da pesquisa de iniciação científica, ainda em andamento, foi apresentada no Congresso de Iniciação Científica da Universidade Federal de São Carlos, realizado em São Carlos, SP de 14 a 18 de outubro de 2013. Poellnitz JC Von (IC), Silva CR (O). Atividades e recursos na formação do terapeuta ocupacional. In: $21^{\circ}$ Congresso de Iniciação Científica da UFSCar. Anais de eventos da UFSCar. São Carlos, SP; 2013. v.10, p.248.

1.Terapeuta Ocupacional, Mestre e Doutora em Educação. Prof ${ }^{a}$ Dr $^{a}$ Adjunta do Departamento de Terapia Ocupacional da Universidade Federal de São Carlos - UFSCar. São Carlos - SP, Brasil. E-mail: carlars@ufscar.br. 2.Terapeuta Ocupacional formada pela Universidade Federal de São Carlos.

Endereço para correspondência: Universidade Federal de São Carlos - UFSCar. Departamento de Terapia Ocupacional. Laboratório de Ensino, Pesquisa e Extensão Atividades Humanas e Terapia Ocupacional - AHTO. Rodovia Washington Luís, km 235, São Carlos, SP, Brasil. CEP: 13565-905. Tel. (16) 3351-8743 / 99733-8567.
} 
s atividades na terapia ocupacional são
compreendidas por meio de diferentes
concepções que por sua vez, concebem os indivíduos e suas ações de forma singular'1, elas são gestadas e constituídas de acordo com momentos históricos, econômicos e políticos.

A história da terapia ocupacional está marcada pela emergência e predominância de modelos assumidos em decorrência das pressões da área médica, de saberes e tecnologias em disputas hegemônicas ${ }^{2}$.

"O relato da história do uso da ocupação terapêutica desde a antiguidade até os dias atuais (...) é tido como uma leitura histórica reducionista da realidade que respaldou os modelos de Terapia Ocupacional do primeiro mundo, nessa fase da globalização da economia capitalista mundial" (p.4) $)^{3}$.

A constituição histórica do papel do terapeuta ocupacional relaciona-se às contraditórias condições da realidade social, assim é necessário compreender em que sociedade esta prática social foi engendrada, suas conjunturas político-econômicas que dimensionaram e caracterizaram seu papel técnico-político ${ }^{4}$.

Para Lopes ${ }^{5}$, o debate sobre o surgimento da terapia ocupacional correlaciona-se às questões ligadas à formação de profissionais da saúde e o processo de especialização que ocorreu com maior intensidade, a partir do século XIX, tendo como panorama a Revolução Industrial.

A Primeira Guerra Mundial foi um importante marco, pois principalmente nos EUA, houve um movimento de "reconstrução nacional" para a retomada do desenvolvimento, readaptação e reabilitação física e moral ${ }^{5}$. Além disso, devido à necessidade de serviços de reabilitação profissional foram elementos que contribuíram para o fomento da profissão ${ }^{3}$.

Os primeiros cursos de terapia ocupacional são posteriores à $1^{\text {a }}$ Guerra Mundial, nos Estados Unidos, em 1917, “conduzidos e orientados por médicos com auxílio de enfermeiras e assistentes sociais, que muitas vezes acabaram se transformando em terapeutas ocupacionais" (p. 27) .

Essa característica foi análoga ao surgimento da formação de terapeutas ocupacionais no Brasil, a partir de 1950. Lopes ${ }^{5}$ afirma que o eixo valorizado foi predominantemente direcionado ao 'vocacional' e o 'missionário'.

Em 1963, o Ministério de Educação e Cultura ${ }^{(1)}$ propôs que as matérias básicas para a formação de cursos técnicos, como o de terapia ocupacional, deveriam ser resumidas ao indispensável à compreensão e boa execução dos atos terapêuticos, por meio de um currículo mínimo (2160 horas) realizado em três anos ${ }^{5}$. O Decreto-Lei n ${ }^{\circ} 938$ de 1969 regulamenta a profissão como de 'nível superior'.

A formalização dos cursos em terapia ocupacional decorreu de uma política econômica para expandir e modernizar o processo de produção capitalista e o modelo de atuação esteve fortemente marcado pela perspectiva do Movimento de Reabilitação ${ }^{(2)} \mathrm{Na}$ década de 1970 , os terapeutas ocupacionais iniciam processos e debates para a reformulação do currículo mínimo, pois havia um descompasso entre o ensino e a realidade de saúde ${ }^{5}$. Considerando os padrões internacionais preconizados pela Federação Mundial de Terapia Ocupacional (World Federation of Occupational Therapists), as Comissões de Ensino da Associação Brasileira de Fisioterapia (ABF) e da Associação de Terapeutas Ocupacionais do Brasil (ATOB) promoveram debates e reivindicaram uma possível modificação dos currículos ${ }^{7}$.

O novo currículo mínimo ${ }^{(3)}$ foi aprovado, pelo Parecer $n^{\circ}$. 622/82 do CFE, em 1982, com 3240 horas ministradas entre quatro e oito anos, estabelecendo em específico, o ensino de atividades e recursos - Atividades e Recursos Terapêuticos e Métodos de Instrução, Análise de Atividades Artesanais, Artísticas, Domésticas, Lúdicas, Culturais, Profissionais e Atividades da Vida Diária ${ }^{7}$.

Em 1990, os terapeutas ocupacionais, em diálogo constante com as Comissões de Especialistas de Ensino de Terapia Ocupacional da Secretaria de Ensino Superior - $\mathrm{SESu}^{5}$, buscaram definir seus princípios sobre a formação profissional e apesar dos entraves encontrados, foram construídos instrumentos avaliativos definidos e referendados pela categoria organizada ${ }^{8}$.

A Resolução CNE/CES 6 (2002), do Conselho Nacional de Educação e Câmara de Educação Superior, instituiu as Diretrizes Curriculares Nacionais do Curso de Graduação em Terapia Ocupacional que definiram os

(1) Parecer n $n^{\circ}$ 388/63 do Conselho Federal de Educação - CFE, homologado pela Portaria n $n^{\circ}$ 511/64 de 1963.

(2) Movimento social que surgiu no pós $2^{\mathrm{a}}$. Guerra Mundial e estendeu sua prática para os sistemas de saúde dos países participantes da guerra, para atendimento dos veteranos de guerra, acidentados de trabalho e demais incapacitados, promovendo a capacitação especializada de profissionais para atuarem neste campo (Medeiros, 2011).

(3) A formalização do Currículo Mínimo, nos cursos de graduação do Ensino Superior do país, foi extinta com a Lei de Diretrizes e Bases da Educação de 1996 (Brasil, Lei 9.394 de 20 de dezembro de 1996. 
conteúdos essenciais que contemplavam os fundamentos da profissão, as atividades e recursos, cinesiologia, cinesioterapia, ergonomia, processos saúde-doença, planejamento e gestão de serviços, estudos de grupos e instituições e diferentes áreas de atuação?.

É necessário ressaltar que toda política para a educação superior no Brasil, a partir de 1990, tem relação orgânica com a Reforma do Estado que obedece a lógica neoliberal respondendo aos organismos financiadores internacionais, é a partir desta concepção que devemos compreender as mudanças na abertura de novos cursos, nos padrões de qualidade e avaliações, a flexibilização entre o privado e o público, a redução progressiva de fundos públicos e o aumento das fontes de financiamento, entre outros $^{10}$.

Há grande pressão, em nível internacional - veiculada inclusive através de mecanismos de financiamento (ou não) de projetos por organismos como o Banco Mundial, (...), no sentido de que esses recursos humanos [incluindo terapeutas ocupacionais], nos países periféricos da economia hoje globalizada, tenham, em sua maioria (do ponto de vista do ensino de massas), competência fundamentalmente técnica, e, ainda assim, apenas restrita ao âmbito da reprodução de conhecimentos já estabelecidos, de forma a não gerar condições adequadas para a independência científico-tecnológica-econômica plena dos países em desenvolvimento, pensados, pela ótica dos países centrais, como sendo, em larga medida, reserva de mercado para si próprios (p. 9) ${ }^{7}$.

Nos anos 2000, destacaram-se a institucionalização de uma reforma universitária, sobretudo com a Lei N. ${ }^{\circ}$ 10.861, de 2004 que cria o Sistema de Avaliação da Educação Superior-SINAES ${ }^{5}$ a Lei N. ${ }^{\circ} 11.096$, de 2005 que institui o Programa Universidade para Todos - PROUNI e o decreto N. ${ }^{\circ} 6.096$ de 2007 que institui o Programa de Apoio a Planos de Reestruturação e Expansão das Universidades Federais - REUNI.

Outros balizadores importantes para os cursos de terapia ocupacional são os Padrões Mínimos estabelecidos pela Federação Mundial de Terapeutas Ocupacionais, revisados em $2002^{11}$.

Ressalta-se a importância do ensino de atividades na formação do terapeuta ocupacional, uma vez que são consideradas centrais nos diferentes processos terapêuticos ocupacionais, contemplando sua multiplicidade de campos e populações. A riqueza da abordagem da terapia ocupacional está exatamente nesta possibilidade de conectar vários saberes e resultar numa prática interdisciplinar quase sempre $^{12}$.

A formação deve capacitar para o acompanhamento sensível de sujeitos, grupos e coletivos, assim como, de suas diversidades e adversidades, para a compreensão, mediação e resolução de suas demandas. Representando, assim, "oportunidades de encontro e diálogo entre os diferentes indivíduos da sociedade e possibilitando a emergência de produções significativas e desalienadoras, que envolvem um sujeito inserido em determinado tempo e espaço" (p.45)

Ressalta-se a estratégia acerca da experimentação da atividade, como prática essencial para a criação de habilidades, competências e atitudes particularizadas pelo terapeuta ocupacional, introjetadas pelas vivências em si. "A experimentação deve estar presente na formação do terapeuta ocupacional [...] proporcionando amadurecimento gradual e construção da identidade" (p.135) ${ }^{14}$.

O ensino de atividades na terapia ocupacional, inicia um processo de construção profissional fornecendo espaço para expressão de sentimentos, ações, práticas e reflexões ${ }^{15}$. "A atividade humana é relacional e pode instaurar aprendizagens de si, do outro, de técnicas, instrumentos e territórios, facilitando novas experiências relacionais que enriquece e aumenta a potência de ação e criação da existência" (p.36) ${ }^{16}$.

\section{OBJETIVOS E MÉTODOS}

A pesquisa " $\mathrm{O}$ ensino das atividades e a formação do terapeuta ocupacional" foi realizada entre julho de 2012 a agosto de 2013, com financiamento do Conselho Nacional de Desenvolvimento Científico e Tecnológico (CNPq). Seu objetivo foi de mapear e analisar as propostas de ensino de atividades e recursos nos cursos de terapia ocupacional de Instituições de Ensino Superior (IES) públicas, do Estado de São Paulo, sendo elas: Universidade de São Paulo USP - campus São Paulo; Universidade de São Paulo USP campus Ribeirão Preto; Universidade Federal de São Carlos UFSCar - campus São Carlos; Universidade Federal de São Paulo UNIFESP - campus Baixada Santista e; Universidade Estadual Paulista Júlio de Mesquita Filho UNESP- campus Marília. Estes cursos representavam, no período da pesquisa, $10 \%$ do total em âmbito nacional, ou ainda, $25 \%$ do total de cursos públicos na área ${ }^{(4)}$.

(4) O Brasil contava com 49 cursos de Terapia Ocupacional em $2013^{17}$. Destes, $60 \%$ são realizados por IES privadas e $40 \%$ por IES públicas, o Estado de São Paulo concentra 33\% do total de cursos, sendo que, 69\% (11 cursos) são oferecidos em IES privadas e $31 \%$ (5 cursos) estão em IES públicas. 
Os procedimentos metodológicos utilizados foram:

- Levantamento e análise dos Projetos Políticos Pedagógicos e das disciplinas específicas para o ensino de atividades e recursos (ementa, período de oferta, conteúdo, duração e carga horária);

- Realização de entrevistas com coordenadores dos cursos de graduação em terapia ocupacional, a partir de roteiro elaborado para tal finalidade, que contemplava: informações pessoais, sobre a IES, infraestrutura para o ensino da temática e informações adicionais. O coordenador após a entrevista indicava professores para participar da pesquisa, considerando aqueles que ministravam disciplinas específicas de atividades e recursos, no total foram indicados 24 docentes;

- Aplicação de questionário destinado aos docentes que após o aceite da Carta Convite enviada para seu correio eletônico, responderam um questionário composto por questões abertas e fechadas, estruturado pelos itens: dados pessoais, formação acadêmica e complementar, carreira docente, disciplinas específicas de atividades e recursos (conteúdo, estratégias pedagógicas, avaliações, infraestrutura dos laboratórios de ensino, referenciais teóricos), atividades de extensão e pesquisa e espaço para informações complementares.

A seguir, as IES com respectivo número de docentes indicados e número de docentes colaboradores: USP campus São Paulo (6/5); USP campus Ribeirão Preto (3/2); UFSCar (5/2); UNIFESP (7/7) e UNESP (3/1).

\section{Tabulação e sistematização dos dados}

As entrevistas foram gravadas e transcritas, os dados quantitativos foram tabulados e produzidos gráficos e tabelas e os dados qualitativos foram agrupados por categorias e elaboradas análises de conteúdo.

Concebemos as atividades e os recursos terapêuticos ocupacionais imprescindíveis na formação, seu ensino ocorre de forma transversal em diferentes momentos da formação, como nas disciplinas práticas e estágios profissionalizantes. Contudo, o foco deste estudo foram as disciplinas consideradas pelos coordenadores como específicas deste campo.

Todos os procedimentos éticos foram respeitados, os colaboradores autorizaram suas participações anuindo os Termos de Consentimento Livre e Esclarecido ${ }^{(5)}$.

\section{RESULTADOS}

Os resultados serão apresentados a partir de categorias de análise, sendo elas: perfil das IES, disciplinas consideradas específicas, carga horária; perfil dos docentes; avaliação dos laboratórios de ensino; processos avaliativos; ensino, pesquisa e extensão e questões identificadas pelos coordenadores.

- Perfil das Instituições de Ensino Superior e disciplinas específicas

O curso de Terapia Ocupacional da Universidade de São Paulo - campus São Paulo foi pioneiro no país e surgiu em 1958. Atualmente, o curso oferece 25 vagas anuais, ministrado em oito semestres, em período integral, total de 3915 horas, possui 15 professores e 15 técnicos terapeutas ocupacionais.

Possui um laboratório de ensino que acomoda as 11 disciplinas de atividades e recursos, sendo elas (60h): Atividades e Recursos Terapêuticos (ART): repertório de atividades, ART: cotidiano, Cinesiologia Aplicada à TO, ART: processos criativos, ART: linguagens, Abordagens Grupais Aplicadas à TO, (45h) ART: atividade lúdica, (30h) TO e as Práticas Corporais I, II e III (30h) e ART: recursos tecnológicos.

O curso de graduação em Terapia Ocupacional, da Faculdade de Medicina de Ribeirão Preto (FMRP), da Universidade de São Paulo, foi criado em $2002 \mathrm{em}$ período vespertino-noturno, com 20 vagas anuais ofertadas, cinco anos de duração e possui dez docentes e três técnicos terapeutas ocupacionais.

Apresenta 14 disciplinas específicas: (60h) Análise da ART Lúdicos e do Lazer, (45h) ART e Ações Humanas, Processos Criativos, Comunicação e TO, ART de Comunicação e Expressão, Psicomotricidade Aplicada à TO, Dinâmica e Abordagens Grupais e TO Análise de Atividades e Recursos Comunitários, (30h) ART e Cotidiano, Cinesiologia e Biomecânica Aplicada à TO, ART Artesanais, Cinesiologia e Biomecânica Aplicada à TO II, Recursos Terapêuticos e Abordagens Corporais em TO, Tecnologia Assistiva, Recursos Adaptativos e TO e TO, Prótese e Órtese.

Possui cinco laboratórios de ensino: Atividades e Recursos Terapêuticos, Expressão Corporal, Multidisciplinar, Terapia Ocupacional na Infância e Adolescência e de recursos lúdicos e de lazer no Centro Integrado de Reabilitação.

O curso de Terapia Ocupacional da Universidade Federal de São Carlos teve início em 1978 e sua última

(5) Pesquisa aprovada pelo Comitê de Ética em Pesquisas com Seres Humanos da UFSCar, sob o parecer 185.502 em 11/12/2012 e Relatório Final aprovado sob o parecer 428.513, em 8/10/2013. 
reformulação curricular é de 2008, o novo currículo adota uma abordagem sócio construtivista da educação ${ }^{18}$.

O curso é integral e oferta 41 vagas anuais através do Exame Nacional do Ensino Médio/Sistema de Seleção Unificada (ENEM/SiSU), sendo uma vaga para indígena ${ }^{18}$. Segundo a coordenadora, desde 2011 a formação é realizada em cinco anos, o corpo docente é formado por 17 professores efetivos e 12 substitutos. A IES possui cinco laboratórios de ensino: Abordagens Corporais, Atividades Plásticas, Atividades Artesanais, Cinesiologia e Órteses e Tecnologias e apresenta seis disciplinas específicas: (60h) Unidade Educacional de Recursos e Atividades (UEREATO) I, II, III, IV, VI e VIII e (90h) UEREATO V e VI.

O curso de Terapia Ocupacional da Universidade Federal de São Paulo - campus Baixada Santista teve início em 2006 e conta com Projeto Político Pedagógico de "caráter interprofissional e interdisciplinar com vistas à integralidade do cuidado" (p.37) ${ }^{19}$.

O curso oferece 40 vagas anuais, por intermédio do ENEM SiSU, ministrado em período integral, durante quatro anos e dispõe de 15 professores. A IES tem quatro laboratórios de ensino: Atividade e Recursos Terapêuticos, Atividades de Vida Diária, Cinesiologia e Atividades de Expressão Corporal.
Possui nove disciplinas específicas à temática: (120h) ART I: cotidiano e repertório de atividades, (80h) ART II: atividade de vida diária, ART: processos criativos e de comunicação verbal e não verbal, ART: atividades lúdicas e de lazer, TO em Saúde e Trabalho, ART: tecnologia assistiva, órtese, prótese e adaptação, (60h) Abordagens Teóricas e Tendências Contemporâneas em TO, Abordagem Grupal e Estudo do Movimento Humano Aplicado à TO: medidas e avaliação.

O curso de Terapia Ocupacional da Universidade Estadual Paulista Júlio de Mesquita Filho foi implantado em 2003, com oito semestres de duração em período integral e oferta de 40 vagas anuais.

O corpo docente é formado por sete professores efetivos e três substitutos da área ${ }^{(6)}$. A IES possui três laboratórios de ensino: Atividades e Recursos Terapêuticos, Prótese e Órtese e Cinesioterapia e apresenta cinco disciplinas específicas de 60h: Análise e Aplicação da Atividade em TO, Cinesioterapia, Recursos Terapêuticos em TO, Recursos Adaptados na Comunicação Alternativa, Prótese e Órtese.

As disciplinas específicas de atividades e recursos apresentam $11,6 \%$ em média da carga horária total dos cursos nestas IES (Tabela1).

Tabela 1 - Carga Horária das Disciplinas Específicas de Atividades e Recursos

\begin{tabular}{ccccc}
\hline IES & $\begin{array}{c}\mathbf{N}^{\circ} \text {. de disciplinas } \\
\text { específicas }\end{array}$ & CH Total do Curso & CH específica & $\begin{array}{c}\text { Disciplinas específicas no } \\
\text { curso (\%) }\end{array}$ \\
\hline USP SP & 11 & $3915 \mathrm{~h}$ & $465 \mathrm{~h}$ & 12 \\
USP RP & 14 & $3840 \mathrm{~h}$ & $540 \mathrm{~h}$ & 14 \\
UFSCar & 6 & $4300 \mathrm{~h}$ & $420 \mathrm{~h}$ & 9,8 \\
UNIFESP & 9 & $4540 \mathrm{~h}$ & $680 \mathrm{~h}$ & 15 \\
UNESP & 5 & $4125 \mathrm{~h}$ & $300 \mathrm{~h}$ & 7,3 \\
\hline
\end{tabular}

A partir da análise dos projetos pedagógicos, é perceptível que os currículos ofertados se diferenciam entre si, com estruturas particulares que em alguns casos privilegiam determinadas técnicas e conteúdos em detrimento de outros, direcionando forma, conteúdo e aprendizagem, a partir de suas matrizes curriculares, mas também pela influência do corpo docente, que direciona métodos e conteúdos de forma singular.

As Reformas do Estado em relação às políticas educacionais do ensino superior produziram uma série de características presentes nos dados, sobretudo em relação à infraestrutura institucional, tais como, questões relacionadas à sobrecarga de trabalho docente, aliada à falta de docente nos cursos e às necessidades relacionadas às condições de ensino.

(6) Trata-se de contratações temporárias. 
- Perfil dos Docentes

É possível perceber (Gráfico 1) que os docentes da USP - SP atuam na IES desde o início da carreira docente, com o ensino da temática, com uma exceção. Em relação à USP - Ribeirão Preto e à UNIFESP, o tempo de exercício profissional como docente e o tempo ministrando disciplinas relacionadas ao ensino de atividades e recursos é bem próximo, o tempo na instituição é recente, já que se trata de cursos novos e docentes experientes. Já na UFSCar e UNESP verifica-se que o envolvimento dos docentes com a temática teve início com sua inserção na IES.

Gráfico 1 - Docência em cada IES

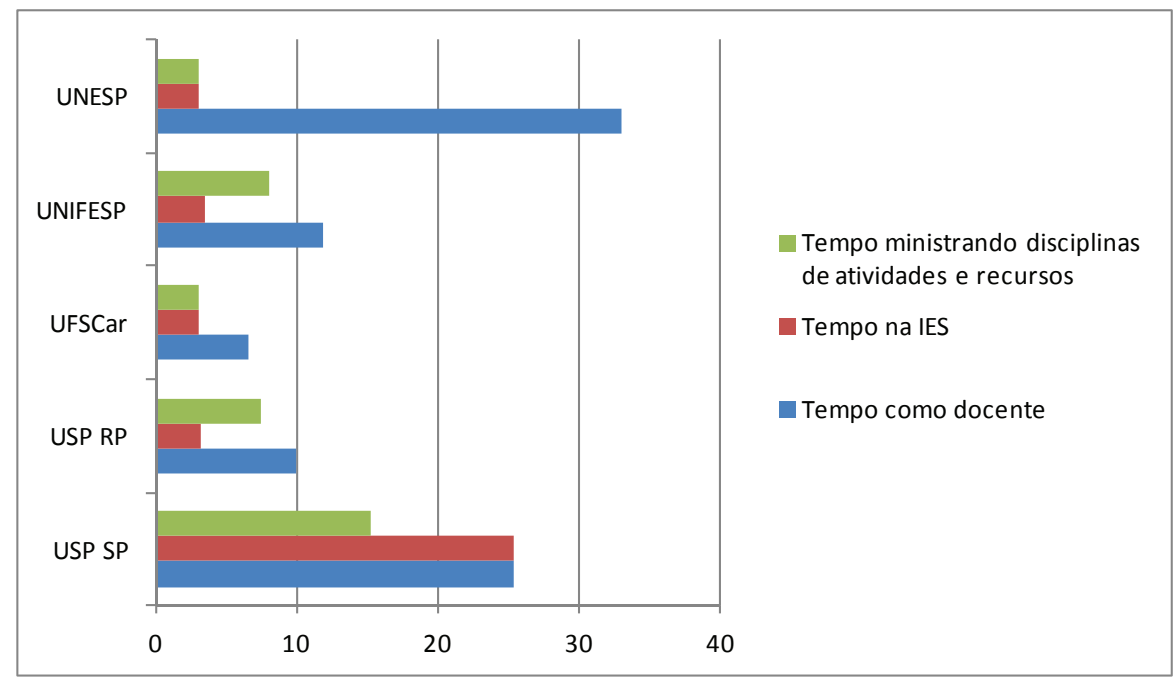

Em relação à formação e às áreas de atuação dos docentes, foi possível perceber a diversidade e pluralidade dos docentes, sendo que a maioria, 64,7\% (11) apresentou mais de uma área. Apenas três docentes $(17,6 \%)$ citaram atividades e recursos como área de atuação (Tabela 2).

Foi possível traçar uma correlação entre a formação dos docentes e sua forma de ensinar atividades na terapia ocupacional, direcionando concepções, métodos e referênciais. Além disso, a formação em outras áreas e aquelas externas à academia parecem contribuir de forma marcante no processo de ensino e aprendizagem.

- Laboratórios de Ensino

No geral, os docentes avaliaram os laboratórios de ensino considerando-os bons, havendo maior insatisfação em relação ao espaço físico, mobiliário, equipamento, falta de apoio e material.

- Processos avaliativos
Seis docentes $(35 \%)$ indicaram realizar entre quatro e cinco processos avaliativos nas disciplinas semestrais voltadas ao ensino de atividades e recursos, os formatos avaliativos variaram, as avaliações processuais e 'trabalhos' $(82 \%)$, seguidos de apresentações orais (76\%), participação (70\%), simulação de casos e avaliação de portfólios (53\%), estudos de casos (47\%), avaliação cognitiva/prova (35\%), dinâmicas de grupo, entre outros (29\%).

- Extensão e pesquisa

Em relação às atividades de pesquisa e extensão universitária, temos que 53\% (9) docentes realizam essa atividade relacionada com a temática, 35\% (6) não oferecem e 6\% (1) oferecem não diretamente. Em relação à atividade de pesquisa, $29 \%$ (5) responderam que realizam de forma integrada à temática e $65 \%$ (11) não realizam ${ }^{(7)}$. O que demonstra envolvimento dos docentes, sobretudo com a extensão universitária vinculada com o ensino e ressalta-se o um número bem menor de docentes com atividades de pesquisa no campo.

(7) Um docente não respondeu às questões relacionadas à extensão e à pesquisa. 
Silva CR, Poellnitz JCV. Atividades na formação do terapeuta ocupacional. Rev Ter Ocup Univ São Paulo. 2015 jan./abr.;26(1):74-82.

Tabela 2 - Áreas e/ou Campos de Atuação em Terapia Ocupacional dos Docentes

\begin{tabular}{|c|c|c|}
\hline IES & Docentes & Áreas de Atuação \\
\hline \multirow{5}{*}{ USP SP } & 1 & Saúde Mental; Social; Interface Arte, Saúde, Cultura \\
\hline & 2 & Arte e Corpo em Terapia Ocupacional \\
\hline & 3 & Deficiência Física e Sensorial \\
\hline & 4 & $\begin{array}{l}\text { Deficiência Intelectual e Transtornos Globais do Desenvolvimento; Atividade } \\
\text { Lúdica, Infância e Adolescência com Deficiência }\end{array}$ \\
\hline & 5 & Social \\
\hline \multirow{2}{*}{ USP RP } & 1 & Infância e Adolescência; Social \\
\hline & 2 & Social \\
\hline \multirow{2}{*}{ UFSCar } & 1 & Disfunção Física Adulto \\
\hline & 2 & Neurologia Infantil \\
\hline \multirow{7}{*}{ UNIFESP } & 1 & Educação; Saúde Mental Infantil \\
\hline & 2 & Disfunção Física; Tecnologia Assistiva \\
\hline & 3 & Generalista \\
\hline & 4 & Gerontologia \\
\hline & 5 & Saúde do Trabalhador; Saúde e Trabalho \\
\hline & 6 & $\begin{array}{l}\text { Disfunção Física; Terapia da Mão; Saúde do Trabalhador; Contextos } \\
\text { Hospitalares }\end{array}$ \\
\hline & 7 & $\begin{array}{l}\text { Saúde Mental; Grupos; Atividades e Recursos Terapêuticos; Atenção Básica; } \\
\text { Ações Territoriais }\end{array}$ \\
\hline UNESP & 1 & Disfunção Física \\
\hline
\end{tabular}

- Avaliação dos coordenadores

Quando questionamos os coordenadores sobre o ensino de atividades e recursos foram trazidas questões complexas sobre a formação. Uma coordenadora afirma a progressão do ensino de tal conteúdo, mas ainda permanece um sentimento de que não houve aprofundamento suficiente e que o ensino das atividades e seus recursos não é homogêneo, pois depende do percurso do discente

"precisa melhorar ainda a estrutura, mas eu acho que é bom o que a gente ensina, apesar dos alunos terem um pouco de dificuldade de aprender na ART aqui e na hora de aplicar as atividades eles se perdem, eles sentem um pouco de dificuldade de ir para a prática [...] se ensina aqui mas não consegue transportar ou [tem] uma dificuldade muito grande de associar a cultura do indivíduo, a história de vida dele, com a necessidade que a atividade vai ter para aquela atuação".

Ainda em relação às dificuldades,

"a dificuldade no ensino da atividade é a percepção do próprio aluno, do que é a atividade [...] tem aluno hoje vindo que não quer nem saber de atividade [...] $e$, a gente acredita que você tem que vivenciar, você tem que aprender a técnica para poder aplicar".

Outra coordenadora apresenta reflexão importante para a temática quando afirma:

"às vezes, fica meio ensino, eu sei que os professores fazem uma reflexão e tal, mas eu acho que falta a 
gente costurar, principalmente com fundamentos da TO, não é a reflexão do uso das atividades, não é a reflexão da análise da atividade, é a reflexão da atividade como parte dos fundamentos da terapia ocupacional".

Além disso, os coordenadores referendaram que tal temática é transversal nos cursos de graduação e está presente não apenas nas disciplinas específicas, mas em diversos momentos da formação.

\section{Considerações Finais}

Foi possível verificar algumas diferenças e outras semelhanças na estrutura curricular, em relação ao ensino de atividades e recursos em terapia ocupacional, a partir de um universo particular - IES públicas no Estado de São Paulo. Verificamos a concentração de determinados conteúdos de atividades e recursos correlacionados àqueles domínios da formação estruturante de cada docente.

\section{REFERÊNCIAS}

1. Silva CR. As atividades como recurso para pesquisa. Cad Ter Ocup UFSCar, São Carlos. 2013;21(3):461-70. doi: http:// dx.doi.org/10.4322\%2Fcto.2013.048.

2. Medeiros MHR. Terapia ocupacional: um enfoque epistemológico e social. São Carlos: EdUFSCar; 2003.

3. Soares LBT. História da Terapia Ocupacional. In: Cavalcanti A, Galvão C. Terapia Ocupacional: fundamentação \& prática. Rio de Janeiro: Guanabara Koogan; 2011. p.3-9.

4. Soares LBT. Terapia ocupacional: lógica do capital ou do trabalho? São Paulo: Hucitec; 1991.

5. Lopes RE. Cidadania, políticas públicas e terapia ocupacional, no contexto das ações de saúde mental e saúde da pessoa portadora de deficiência, no Município de São Paulo [Tese]. Campinas: Universidade Estadual de Campinas; 1999. Disponível em: http://www.bibliotecadigital.unicamp.br/ document $/$ ?code $=$ vtls 000184393 .

6. De Carlo MMRP, Bartalotti CC. Caminhos da Terapia Ocupacional. In: De Carlo MMRP, Bartalotti CC, organizadores. Terapia Ocupacional no Brasil - fundamentos e perspectivas. São Paulo: Plexus; 2001. p.19-40.

7. Lopes RE. A formação do Terapeuta Ocupacional: considerações sobre a trajetória de 50 anos no Brasil. In: $1^{\circ}$ Congresso Norte-Nordeste de Terapia Ocupacional, Fortaleza; 2004. Anais. Fortaleza: Governo do Estado de Fortaleza; 2004.
A composição de um corpo docente com formações distintas, articulados entre si, que possam realizar parcerias voltadas não apenas para o ensino, mas para a integração de atividades extensionistas e de pesquisa, retroalimentando as atividades de ensino e produzindo novos conhecimentos para a terapia ocupacional parece ser um caminho profícuo na qualificação dos cursos que pretendem ofertar uma formação generalista.

Vimos a necessidade da temática das atividades e recursos consolidar-se como campo próprio de construção de saber, pois sua fundamentação é basilar para as práticas do terapeuta ocupacional e deve estar composta por conteúdos de base conceitual estruturante integrado aos conteúdos práticos, técnicos, procedimentos e manejos.

Por fim, enfatiza-se a necessidade de atualização de estudos e pesquisas que possam se debruçar sobre a formação do terapeuta ocupacional, a partir das realidades do ensino superior no país, considerando as inúmeras mudanças no perfil dos cursos de terapia ocupacional das últimas décadas.

p.1-15.

8. Tirado MGA, Almeida MC, Lopes RE, Galheigo SM. Terapia Ocupacional. In: Haddad AE, Pierantoni CR, Ristoff D, Xavier IM, Giolo J, Silva LB, organizadores. A trajetória dos Cursos de Graduação na Saúde de 1991-2004. Brasília: Instituto Nacional de Estudos e Pesquisas Educacionais Anísio Teixeira; 2006. Disponível em: http://www.publicacoes.inep. gov.br/portal/download/455.

9. Brasil. Resolução CNE/CES 6, de 19 de fevereiro de 2002. Diário Oficial da União, Brasília, 4 mar. 2002. Seção 1, p.12 [citado 11 out. 2011]. Disponível em: http://portal.mec.gov. br/cne/arquivos/pdf/CES062002.pdf.

10. Minto LW. A educação da "miséria”: particularidade capitalista e educação superior no Brasil [Tese]. Campinas: Universidade Estadual de Campinas; 2011. Disponível em: http://www. bibliotecadigital.unicamp.br/document/?code $=000836877$.

11. Hahn MS, Lopes RE. Diretrizes para a formação de terapeutas ocupacionais - percursos e perspectivas. Pro-Posições, Campinas. 2003;14(1):121-39. Disponível em: http://www. proposicoes.fe.unicamp.br/proposicoes/textos/40-dossie-hahnms_etal.pdf.

12. Furtado E. Conversando sobre identidade profissional. Rev Ter Ocup Univ São Paulo. 1999;10(2/3):46-8.

13. Castro ED, Lima EMFA, Brunello MIB. Atividades humanas 
Silva CR, Poellnitz JCV. Atividades na formação do terapeuta ocupacional. Rev Ter Ocup Univ São Paulo. 2015 jan./abr.;26(1):74-82.

e Terapia Ocupacional. In: De Carlo MMRP, Bartalotti CC, organizadores. Terapia Ocupacional no Brasil - fundamentos e perspectivas. São Paulo: Plexus; 2001. p.41-59.

14. Cruz DMC, Campos IO. A opinião de estudantes de Terapia ocupacional sobre o processo de sua formação profissional. Cad Ter Ocup UFSCar. 2004;12(2):105-14. Disponível em: http://www.cadernosdeterapiaocupacional.ufscar.br/index. $\mathrm{php} /$ cadernos/article/view/184/141.

15. Galheigo SM, Castro ED. Humanização e cuidado - referenciais para a prática da terapia ocupacional junto à pessoa com câncer. In: Othero MB, organizador. Terapia Ocupacional - práticas em oncologia. São Paulo: Roca; 2010. p.16-46.

16. Almeida IS, Ruas TCB, Oliveira AS, Akashi LT. Dialogando sobre o processo de ensino e aprendizagem de atividades e recursos terapêuticos. Cad Ter Ocup UFSCar, São Carlos. 2002;10(2):129-35. Disponível em: http://www.cadernosdeterapiaocupacional.ufscar.br/index.php/cadernos/article/ view/214/168.

17. Palm RCM. Catálogo latinoamericano de asociaciones, carreras y postgrados de Terapia Ocupacional. Curitiba; 2012.

18. UFSCar. Projeto Político Pedagógico do Curso de Graduação em Terapia Ocupacional. São Carlos; 2011 [citado em 7 out. 2012]. Disponível em: http://www.prograd.ufscar.br/ projetoped/projeto TO.pdf.

19. UNIFESP. Projeto Pedagógico Curso de Terapia Ocupacional. São Paulo; 2011 [citado 8 out. 2012]. Disponível em: http:// www.unifesp.br/prograd/portal/index.php?option=com docman\&task $=$ cat_view\&gid=154\&Itemid $=260$.

Recebido para publicação: 07/02/2014

Aceito para publicação: 25/11/2014 